\title{
Cognitive Restructuring, Behavioral Activation and Cognitive Behavioral Therapy in the Treatment of Adult Depression: A Network Meta-analysis
}

Marketa Ciharova $^{1}$, Toshi A. Furukawa ${ }^{2}$, Orestis Efthimiou ${ }^{3,6}$, Eirini Karyotaki ${ }^{1,4}$, Clara Miguel $^{1}$, Hisashi Noma ${ }^{5}$, Andrea Cipriani ${ }^{6,7}$, Heleen Riper ${ }^{1,8,9,10,11} \&$ Pim Cuijpers ${ }^{1,4}$

${ }^{1}$ Department of Clinical, Neuro- and Developmental Psychology, Amsterdam Public Health Research Institute, Vrije Universiteit Amsterdam, Amsterdam, The Netherlands ${ }^{2}$ Department of Health Promotion and Human Behavior, Kyoto University Graduate School of Medicine/School of Public Health, Kyoto, Japan

${ }^{3}$ Institute of Social and Preventive Medicine, University of Bern, Bern, Switzerland ${ }^{4}$ WHO Collaborating Center for Research and Dissemination of Psychological Interventions ${ }^{5}$ Department of Data Science, The Institute of Statistical Mathematics, Tokyo, Japan ${ }^{6}$ Department of Psychiatry, University of Oxford, Oxford, UK ${ }^{7}$ Oxford Health NHS Foundation Trust, Warneford Hospital, Oxford, UK

${ }^{8}$ Research Unit for Telepsychiatry and e-Mental Health, Department of Clinical Research, University of Southern Denmark, Odense, Denmark

${ }^{9}$ Department of Research and Innovation, GGZ inGeest Specialized Mental Health Care, Amsterdam, The Netherlands

${ }^{10}$ Amsterdam UMC, Vrije Universiteit Amsterdam, Psychiatry, Amsterdam Public Health Research Institute, Amsterdam, The Netherlands ${ }^{10}$ University of Turku, Faculty of Medicine, Finland 
Author note:

No funding was received for this study. The protocol for this study has been published in PROSPERO (CRD42019128587).

Acknowledgements:

OE was supported by Ambizione grant No. 180083 from the Swiss National Science Foundation (SNSF). AC is supported by the National Institute for Health Research (NIHR) Oxford Cognitive Health Clinical Research Facility, by an NIHR Research Professorship (grant RP-2017-08-ST2-006), by the NIHR Oxford and Thames Valley Applied Research Collaboration and by the NIHR Oxford Health Biomedical Research Centre (grant BRC1215-20005). The views expressed are those of the authors and not necessarily those of the UK National Health Service, the NIHR, or the UK Department of Health.

\section{Conflict of interests:}

TAF reports grants and personal fees from Mitsubishi-Tanabe, personal fees from MSD, personal fees from Shionogi, outside the submitted work. In addition, TAF has a patent 2018177688 concerning smartphone CBT apps pending, and intellectual properties for Kokoroapp licensed to Mitsubishi-Tanabe. HN reported personal fees from Boehringer Ingelheim, Kyowa Kirin, ASKA Pharmaceutical, and Toyota Motor Corporation outside the submitted work. AC has received research and consultancy fees from INCiPiT (Italian Network for Paediatric Trials), CARIPLO Foundation and Angelini Pharma, outside the submitted work.

Corresponding author: Marketa Ciharova, MSc, Department of Clinical, Neuro- and Developmental Psychology, Vrije Universiteit Amsterdam, m.ciharova@,vu.nl. 


\begin{abstract}
Objective: To examine if cognitive restructuring (CR), behavioral activation (BA) and cognitive behavioral therapy (CBT) result in differential effects in the treatment of adult depression.

Methods: We extracted randomized controlled trials (RCTs) from a database updated yearly from PubMed, PsycINFO, Embase, and Cochrane Library. Network and pairwise metaanalyses were conducted to investigate the effects of CR, BA, and CBT delivered in face-toface individual format, compared with waiting list (WL) and care as usual (CAU), on adult depression. The primary outcome was standardized mean difference (SMD) in post-treatment depression severity. Tolerability of treatments and depression severity at follow-up were also assessed.
\end{abstract}

Results: A total of 45 studies with 3382 participants were included. There was no evidence of difference in effectiveness between CR, BA and CBT. All three interventions were superior to CAU (SMD 0.57, 95\% confidence interval [CI] [0.08 to 1.07]; 0.52 [0.34 to 0.71]; 0.44 [ 0.28 to 0.60$]$, respectively) and WL (1.20 [0.69 to 1.70$] ; 1.15$ [0.90 to 1.40$] ; 1.07$ [0.87 to 1.26$]$ ). No difference in tolerability was found (risk ratio (RR) versus CAU: 1.01 [0.04 to $22.81], 0.84$ [0.63 to 1.11 ], and 0.96 [0.76 to 1.21$]$, respectively). Meta-regression and sensitivity analyses did not produce material differences.

Conclusions: Results suggest that $\mathrm{CR}$ or $\mathrm{BA}$ alone and their combination (CBT) may be effective interventions in comparison to WL and CAU in the treatment of adult depression. There was no evidence suggesting differences in effectiveness among the three treatments. More research is needed to derive conclusions about the performance of CR.

Key words: Depression, cognitive behavioral therapy, behavioral activation, cognitive restructuring, network meta-analysis 
Public Health Significance Statements: This study suggests that BA may be similarly effective to full CBT in the treatment of adult depression. However, current evidence does not allow to draw conclusions about the performance of $\mathrm{CR}$ alone. 


\section{Introduction}

Understanding how psychotherapies work and what core elements are crucial for their effectiveness has become increasingly important for practice as well as theory in the field of psychotherapy. First of all, it is crucial for applying psychological treatments in an efficient manner, thus helping their scalability (Cuijpers, 2017), for uncovering underlying mechanisms of depression (Whisman, 1993), and for optimizing treatments for depression (Cuijpers, Cristea, et al., 2019). In addition, identifying key elements of psychosocial interventions is one of the main steps towards successful implementation of evidence-based psychosocial interventions into clinical practice according to the US Institute of Medicine (England et al., 2015). This also helps to understand the role of common and specific factors in psychotherapy. According to the common-factor model, specific ingredients of psychotherapy produce expectations and create healthy actions, no matter which particular techniques are used. On the contrary, advocates of the specific-factor model propose that specific ingredients remediate a distinct psychological deficit and thus some interventions will be more effective than others thanks to the application of more effective specific ingredients (Wampold \& Imel, 2015).

Cognitive behavior therapy (CBT) is by far the most studied psychotherapy for depression. CBT has been shown to be effective in treating adult depression, at least as effective as any other type of psychotherapy (Cuijpers et al., in press) and pharmacotherapy (Cuijpers, Noma et al., 2020). The most common techniques used in this treatment are cognitive restructuring ( $\mathrm{CR}$; focused on evaluating, challenging, and modifying a client's dysfunctional belief) and behavioral activation (BA; increasing positive interactions between a person and their environment; Mazzucchelli et al., 2009). CR and BA may be applied in a combination (i.e., full CBT), or in separate psychotherapeutic frameworks based on the 
theoretical postulates of active change mechanisms of the respective approaches (Beck et al., 1979; Lewinsohn, 1974).

In order to use CBT in a more efficient way, to implement it successfully into practice and to understand the role of common and specific factors in this treatment, it is necessary to assess whether the combination of $\mathrm{CR}$ and BA, i.e., the full CBT, results in better outcomes for depression as compared to administering cognitive restructuring (CR) or behavioral activation (BA) alone. Nevertheless, evidence from randomized controlled trials (RCTs) comparing these treatments directly provided mixed results. Some trials found no evidence of difference between CBT and BA (Jacobson et al., 1996; Richards et al., 2016), while others found both CR and CBT to be effective for depressive symptoms but did not find evidence for BA (McNamara \& Horan, 1986). Yet, other studies reported no differences between CR and BA, but higher effectiveness of CBT (Taylor \& Marshall, 1977). Furthermore, metaanalyses which focused on these components of CBT presented evidence that both CBT (Cuijpers et al., 2013) and BA (Ekers et al., 2014) were effective treatments for adult depression in general, but not sufficient evidence was reported for adults with noncommunicable diseases (Uphoff et al., 2020). In some meta-analyses, CBT was more effective than CR alone (Cuijpers, Cristea et al., 2019), but others reported no difference between CR and BA, as well as between CBT and BA (Mazzucchelli et al., 2009). Whereas these meta-analyses contributed to the body of research comparing CR, BA, CBT and control conditions, they examined the question using conventional pairwise meta-analyses, which only compare two conditions at a time. Hence, they are restricted by limited available comparisons (Harrer et al., 2019).

To compare all conditions simultaneously in a single analysis, we need to use network meta-analysis (Efthimiou et al., 2016; Furukawa et al., in press). In network meta-analysis, direct comparisons of treatments $\mathrm{A}$ and $\mathrm{B}$ are combined with indirect evidence derived from 
studies which compare these treatments with a common comparator $\mathrm{C}$ (either a control condition or another psychotherapeutic intervention), and thus, network meta-analysis allows the relative effectiveness of all included conditions to be assessed. To the best of our knowledge, the only existing network meta-analysis distinguishing differential effects of CBT and BA (Cuijpers et al., in press), which reported no evidence of differences in effectiveness between these two treatments, considered CBT and CR one condition, and therefore could not make the comparisons among CR, BA, and CBT in the treatment of adult depression in particular. In order to integrate all available evidence from RCTs, we aimed to investigate the effects of $\mathrm{CR}, \mathrm{BA}$, and their combination, compared with control conditions, in individual, face-to-face treatment of adult depression through network meta-analysis, regardless of whether they have been compared in trials in a head-to-head fashion.

\section{Methods}

The protocol for this study has been published in PROSPERO (CRD42019128587; https://www.crd.york.ac.uk/prospero/display_record.php?RecordID=128587). We followed the PRISMA guideline in presenting our study.

\section{Search strategy}

Studies included in this meta-analysis were retrieved from an existing database of randomized controlled trials on psychological treatment for depression which has been described in detail elsewhere (Cuijpers, Karyotaki et al., 2020; Cuijpers, Karyotaki, Harrer, \& Ebert, 2019). This database was created through a comprehensive literature search by two researchers independently in four major bibliographical databases (PubMed, PsycINFO, Embase and the Cochrane Library), by combination of both index terms and text words indicative of depression and psychotherapy, and applying filters for RCTs (the full search 
string for PubMed is provided in Appendix A). Further searches were conducted in several bibliographical databases established in non-Western countries (Cuijpers, Karyotaki, Reijnders, Purgato, \& Barbui, 2018). References of previous meta-analyses focused on psychological treatments of depression were also examined. The database currently involves 720 studies from 1966 to 1 January 2020, is updated yearly and has been previously used in a series of meta-analyses (Cuijpers, 2017). Our search was not restricted to any language. Titles and abstracts of all records present in the database were examined by 2 independent researchers and studies possibly meeting inclusion criteria were retrieved as full-text and inspected. The decision about inclusion was made by 2 independent researchers. All disagreements were solved by discussion.

\section{Identification and Selection of Studies}

Studies were included if (1) they reported on an RCT; (2) at least two of the following three conditions were compared: $\mathrm{CR}, \mathrm{BA}$, their combination into $\mathrm{CBT}$, to each other, to care-asusual (CAU) or to waiting list condition (WL); (3) it was performed in adults (> 18 years); (4) the participants had a diagnosis of depression (based on a clinical interview and/or cut-off on a self-report measure); and (5) they reported a measure of depressive symptoms at a posttreatment assessment.

Appendix B involves definitions of all included interventions. In order to reduce clinical heterogeneity, we only included studies in which interventions were delivered in an individual face-to-face format. Studies on group, telephone, guided or unguided self-help (internet-based or through other media, such as a book) were excluded. Control groups could receive CAU or stay on WL. Participants were categorized as being in CAU if they had access to regular routine care. If no intervention was provided, it was assumed that 
participants had access to routine care and were also categorized as CAU. If a group received CAU while waiting to receive the intervention, it was considered WL.

Studies were excluded if they did not report sufficient statistics to perform the metaanalysis, i.e., means and standard deviations (SD), standard errors, or confidence intervals of means $(95 \% \mathrm{CI})$. Comorbid general medical or psychiatric disorders were not used as an exclusion criterion and patients could come from any setting (primary care, specialized mental health care, general medical hospitals) or could be recruited through the community or other methods, such as systematic screening. We excluded studies that involved patients with comorbid substance use disorders, and studies on inpatients, because the effect sizes of psychotherapy have been found to differ from those in outpatients, probably because the usual care in inpatient settings differs considerably (Cuijpers et al., 2011).

\section{Quality assessment}

Risk of bias of included studies was assessed as low, or as high/unclear (i.e., a study does not provide enough information) by two independent researchers using four criteria of the tool from the Cochrane Collaboration (Higgins \& Green, 2011): (1) adequate generation of a randomized sequence; (2) adequate concealment of allocations prior to assignment; (3) masking of outcome data assessors; (4) adequate handling of incomplete outcome data, which was rated as good quality if intention-to-treat (ITT) analyses were applied. Disagreements were resolved through discussion.

\section{Data extraction}

We extracted characteristics of the study (year of publication, country, outcome), participants (definition of depression, target group, recruitment method, sample size, size of the compared groups), psychological intervention (type of intervention, type of BA, type of manual used for 
cognitive restructuring, other techniques used next to CR and BA, number of sessions), type of control, and data (means, SDs, and tolerability) at post-treatment and at follow-up (means, SDs). When available, ITT data were preferred above completers-only data.

\section{Outcomes}

In case a study reported on multiple outcome measures indicating severity of depression, we used an algorithm, previously reported in several studies (e.g., Cuijpers, Noma et al., 2019), in order to select the most reliable one. The current approach allowed us to retrieve the most reliable information per study, while allowing us to use all the eligible studies regardless of whether they were using a clinician- or self-rated instrument, thus without reducing the statistical power of the analysis. The priority was given to Hamilton Rating Scale for Depression (Hamilton, 1986), followed by Beck Depression Inventory I or II (Beck et al.,1961; Beck et al., 1996), another clinician-rated instrument, another self-report instrument with the priority for the Patient Health Questionnaire-9 (Kroenke et al., 2001), the Center for Epidemiologic Studies-Depression Scale (Radloff, 1977), the Hospital Anxiety and Depression Scale-Depression (Zigmond \& Snaith, 1983), or any other instrument.

Primary outcome was calculated at post-treatment, as the difference between conditions after the end of the treatment. Results at 3 to 12-month follow-up were also investigated for the studies that reported such findings. Study dropout for any reason during the acute-phase treatment served as operationalization of tolerability of different interventions.

\section{Statistical Analysis}

First, we conducted pairwise meta-analyses for all direct comparisons using a random-effects model. The standardized mean difference (SMD) was used for continuous (depression 
severity) and relative risk (RR) for binary (tolerability) outcomes. The SMD was calculated as Cohen's $d$, where a positive (negative) SMD in the comparison of $\mathrm{X}$ versus $\mathrm{Y}$ indicates that $\mathrm{X}(\mathrm{Y})$ is a better treatment. We considered a Cohen's $d$ of 0.20 to be small, of 0.50 moderate, and of 0.80 large (Cohen, 1988). We calculated $I^{2}$ statistic to assess the homogeneity of effect sizes. A value of $0 \%$ indicates no observed heterogeneity, and we assumed an of $25 \%$ to indicate low heterogeneity, $50 \%$ moderate and $75 \%$ high heterogeneity (Higgins et al., 2003). We also computed the 95\% CI around the $I^{2}$-statistic (Ioannidis et al., 2007) with the non-central $\chi^{2}$-based approach in the Heterogi module for Stata (StataCorp; Orsini et al., 2006). We examined the possibility of publication bias and small study effects (PB-SSE) by visually inspecting the contour enhanced funnel plots for any signs of asymmetry. We also conducted Egger's test (Egger et al., 1997) for comparisons with 10 studies or more, for the efficacy outcome. We created forest plots of comparisons based on 5 or more studies, and funnel plots of comparisons based on 10 or more studies.

Next, we used network meta-analysis to combine direct and indirect evidence for all relative treatment effects (Mavridis, 2019). An important assumption of network metaanalysis is that of transitivity (Cipriani et al., 2013), i.e., that the distribution of effect modifiers is similar across treatment comparisons. To assess this assumption, we summarized important study and patient characteristics (type of recruitment, diagnosis, target group, risk of bias, whether the country was western or non-western, baseline severity of depressive symptoms) after grouping the studies according to the treatments they compared. Then, we checked for important discrepancies in their distribution across the comparisons.

The geometry of the network evidence was summarized by a network plot (Hutton et al., 2015). We applied a random-effects network meta-analysis model (Salanti et al., 2008) due to the expected clinical and methodological heterogeneity of treatment effects among included studies (Salanti, 2012). We fitted the model in a frequentist setting and calculated 
95\% CIs and 95\% prediction intervals (PrI; the interval within which the true treatment effect of a future study is expected to lie). We assessed the ranking of interventions using the surface under the cumulative ranking curve (SUCRA; Hutton et al., 2015; Salanti et al., 2011). We also created a contribution plot, which shows the percentage of contributions from the direct comparisons for the mixed and indirect estimates. To inspect the consistency of the network, we used the loop inconsistency approach for testing inconsistency locally for all loops in the network (Chaimani et al., 2013). We used the design-by-treatment approach to test inconsistency globally in the network (Higgins et al., 2012).

Meta-regression analyses were performed for pairwise comparisons using type of recruitment (community, clinical or other), type of diagnosis (studies in which participants met criteria for a depressive disorder versus studies where participants scored high on a selfreport scale), target group (adults in general versus specific target groups), publication year (continuous variable), whether the study was conducted in a western or non-western country, and risk of bias (continuous variable). Sensitivity analyses were conducted by analyzing only studies evaluated at low risk of bias, only with studies where Beck's (1979 or 1995) manual was used, and with studies in which a specific type of behavioral activation (pleasant activities, self-control, contextual or behavioral activation treatment for depression) was used (Mazzucchelli et al., 2009). In addition, some studies (i.e., Euteneuer et al., 2017; Pecheur \& Edwards, 1984; Propst et al., 1992; and Rief et al., 2018) reported results on two or more arms of the same type of psychotherapy, but with slight modifications (for example, with more emphasis on religious metaphors in $\mathrm{CR}$, or physical exercise or mindful sensory perception in BA). Hence, we performed the main meta-analyses pooling these conditions together so that each study had only one outcome for one type of therapy. Subsequently, we conducted a sensitivity analysis where these conditions were considered separate psychotherapies. 
Analyses were conducted in Stata/SE, version 16.1 for Mac (StataCorp., 2019; White, 2015). Following recent recommendations (Efthimiou \& White, 2020), we avoided using the concept of "statistical significance" and we concentrated on interpreting the estimated effect sizes and confidence intervals for all our analyses.

\section{Results}

\section{Selection, Inclusion, and Characteristics of Studies}

As shown in the PRISMA flowchart (Appendix C), which describes the process of inclusion as well as the reasons for exclusion, a total of 24647 abstracts (18217 excluding duplicates) were examined and 2912 full texts were retrieved for further inspection. In the end, 2867 studies were excluded and 45 studies with 3382 participants met the inclusion criteria. Characteristics of included studies are given in Appendix D, while Appendix E contains references of the studies.

The 45 included studies reported results of 32 CBT, 4 CR, 19 BA, 20 CAU and 19 WL conditions. While in 28 studies $(62.2 \%)$ participants met diagnostic criteria for a mood disorder, participants in 17 studies (37.8\%) scored above a cut-off point of a self-report instrument. Nineteen studies $(42.2 \%)$ used community means of recruitment, 14 studies (31.1\%) recruited solely clinical samples and 12 studies $(26.7 \%)$ used other means of recruitment. The target group consisted of unselected adults in $19(42.2 \%)$ studies, both student and elderly participant samples in $6(13.3 \%)$ studies, samples with comorbid general medical disorder in $9(20.0 \%)$ studies, $1(2.2 \%)$ study focused on women with postpartum depression and $4(8.9 \%)$ on other specific target groups. A great majority of studies (39; 86.7\%) was conducted in Western countries. As for other techniques used besides CR and BA, the authors listed problem-solving techniques in $9(20.0 \%)$ studies, physical activity as part of BA, relaxation (each in 3 studies; 6.7\%), mindful sensory tasks as part of BA, 
religious rationale in $\mathrm{CR}$ or providing a booklet to the participant (each in 2 studies; $4.4 \%$ ). Other techniques (family psychoeducation, coping strategies, advice on sleeping problems etc.) were reported in 1 study only. A full overview of reported techniques is given in Appendix D.

Regarding risk of bias, an adequate sequence generation was reported by 23 studies (51.1\%), allocation to conditions by an independent or third party was reported by 19 studies (42.2\%), 20 (44.4\%) studies reported masking of outcome assessors and 18 studies (40.0\%) used self-report measures. ITT analyses were conducted in 28 studies (62.2\%). All quality criteria were met by 12 studies (26.7\%), 19 studies (42.3\%) met 2 or 3 criteria, and 14 studies $(31.1 \%) 1$ or no criterion of quality.

\section{Pairwise Meta-analyses}

Results of pairwise meta-analyses are shown in Table 1. The pairwise meta-analyses involved five comparisons with five or more studies, and forest plots of these comparisons are shown in Appendix F. There was evidence of differences between CBT (1.07 [0.81 to 1.33]) and also BA (1.52 [1.13 to 1.91]) versus WL, both with large effect sizes. The estimated difference between CBT (0.48 [0.28 to 0.68$])$; and BA (0.49 [0.27 to 0.71$])$ versus CAU were small to moderate, favoring $\mathrm{CBT}$ and BA. We also found evidence for difference between CR versus WL in favor of CR with a large effect size (1.84 [0.98 to 2.70]), but there were no studies comparing CR versus CAU. In addition, we found no evidence of differences in effectiveness of CBT versus BA (-0.03 [-0.19 to 0.13]); CBT versus CR (0.75 [-0.01 to $1.51])$; and CR versus BA (0.13 [-0.41 to 0.66$])$.

Heterogeneity was low to moderate ( $I^{2}$ statistic ranged from 0 to $53 \%$ ) in all comparisons, but the $95 \%$ CIs of the $I^{2}$ statistic were wide. Only for one comparison (CBT vs 
WL), there was evidence for PB-SSE (Appendix G). There were two comparisons in which Egger's test could not be applied due to low number of studies.

\section{Network Plot}

Figure 1 shows the network plot, while the number of studies for each comparison is shown in Table 1. The network was well connected. The comparison between CBT and WL was the most examined, while CR was relatively weakly connected to the network. Closed loops were found for all conditions. Appendix $\mathrm{H}$ presents the contribution plot.

\section{Network Meta-analysis}

Table 2 shows the main results of the network meta-analysis. There was evidence of increased effectiveness of CBT (1.07 [0.87 to 1.26]); CR (1.20 [0.69 to 1.70]); and BA (1.15 [0.90 to 1.40$])$ versus WL, with large effect sizes. There was also evidence of superiority compared to CAU, but with small to moderate effect sizes ( 0.44 [ 0.28 to 0.60 ]; 0.57 [0.08 to 1.07]; and 0.52 [0.34 to 0.71 ], respectively). We found no evidence of a difference between CBT, CR and BA. CAU was found to be superior to WL (0.63 [0.38 to 0.87]).

Regarding tolerability, there was little evidence to support superiority of any intervention (CBT, CR, BA) above the others. CBT (0.76 [0.50 to 1.15$] ; 0.96$ [0.76 to 1.21$]$, respectively); CR (0.80 [0.04 to 18.08 ]; 1.01 [0.04 to 22.81 ], respectively); and BA (0.66 [0.42 to 1.05$]$; 0.84 [0.63 to 1.11$]$, respectively) were also not more tolerable than WL or CAU.

Figure 2 presents the estimated effects of all treatments using CAU as the reference and Table 3 shows the ranking of treatment formats (SUCRA).

\section{Assessment of transitivity and inconsistency}


We summarized potential effect modifiers (Appendix I) after grouping studies by treatment comparison. This inspection suggested that these characteristics were distributed across the comparisons in the network evenly, with the exception of the comparison between CR and WL. Both studies performing this comparison only recruited patients from the community and only included participants with lower baseline severity of depressive symptoms. This was in contrast with the rest of the comparisons, raising concerns about the transitivity assumption required for network meta-analysis. In line with this, the loop-specific approach for inconsistency showed that the loop CBT-CR-WL was particularly inconsistent, while there was weaker evidence for the remaining loops (Appendix J). The design-by-treatment interaction test also found some evidence of global inconsistency across all comparisons ( $p$ $=.09)$.

\section{Long-term Effectiveness}

Table 4 presents the results of the network meta-analysis at 3 to 12 months of follow-up. Since 6 out of 10 comparisons were reported in 2 or fewer studies (out of which 4 were not reported in any studies) and only two comparisons (CBT versus CAU and BA versus CAU) had more than 5 studies, the network was not well connected. In addition, only 1 study examined WL. No local or global inconsistency in the network was found by the loopspecific approach or design-by-treatment interaction model $(p=0.67)$. Using network metaanalysis, we found evidence of superiority of CBT (1.13 [0.37 to 1.88$])$; CR (1.44 [0.49 to 2.38]); and BA (1.15 [0.41 to 1.89]) over WL, all with large effect sizes. There was also evidence of superiority over CAU (CBT: 0.37 [0.26 to 0.47]; CR: 0.68 [0.08 to 1.28]; BA: 0.39 [0.26 to 0.52$])$, with small to moderate effect sizes. There was no evidence of difference between CBT, CR and BA, and CAU was superior to WL (0.76 [0.01 to 1.51]). Nevertheless, 
these findings have to be considered with caution, due to small number of comparisons, particularly for WL.

\section{Meta-regression and Sensitivity Analyses}

Appendix $\mathrm{K}$ shows the results of the meta-regression analyses. These analyses showed that some variables were strongly associated with the treatment effect, namely means of recruitment (CBT vs BA), whether diagnostic interview was used to assess if participants were eligible for the study (CBT vs CR), and whether a study was conducted in a western or a non-western country (CBT vs WL, and CBT vs CAU).

Sensitivity analyses are reported in appendices L to Q. The first sensitivity analysis (Appendix L) was limited to low risk of bias studies. All studies reporting on a CR arm were assessed as high-risk studies, therefore, the CR condition was completely removed. Results were similar to the main analysis, with no evidence of differences between CBT and BA, and intervention conditions being superior to WL and CAU. Again, CAU was more effective than WL. The results were comparable to the overall findings also in the second (limited to studies using Beck's manual of CBT; Appendix M), the third (with only the pleasant activities type of BA included; Appendix N), the fourth (including only studies with BA intervention of the contextual type; Appendix O) and the fifth (only studies with BA intervention of the behavioral activation treatment for depression [BATD] type; Appendix P) sensitivity analyses. Nevertheless, when including only the BATD type, the BA intervention was superior to the full CBT with a moderate effect size (0.73 [0.15 to 1.31$])$.

In the sixth sensitivity analysis (Appendix Q), we considered two and more arms in the same study looking into the same type of psychotherapy as different interventions. The results were again comparable to the main analysis, with no evidence of differences between 
CBT, CR and BA, these interventions being superior to CAU and WL, and with WL inferior to CAU.

\section{Discussion}

To the best of our knowledge, the current analysis is the first network meta-analysis comparing CBT, CR and BA simultaneously and it included the largest number of studies focused on the comparisons of interest. The aim of the current study was to examine the comparative effects of $\mathrm{CR}, \mathrm{BA}$, their combination as $\mathrm{CBT}$, and control conditions in the treatment of depression in adults. We did not find evidence of differences between CBT, CR and BA. Compared to CAU, the effect sizes of CBT, CR and BA were moderate, while they were large in comparison to WL. CAU was more effective than WL. CR alone was only examined by trials with high risk of bias and thus no firm conclusions can be drawn about its performance. Similar results were obtained at long-term, however, these findings must be considered with caution due to low number of studies. We also did not find evidence of differences in tolerability of the conditions.

The results of the current study are in line with findings of some of the previous studies (Cuijpers et al., in press; Jacobson et al., 1996; Mazzucchelli et al., 2009; Richards et al., 2016) and may also be in agreement with the common-factor model of psychotherapeutic effects (Wampold \& Imel, 2015). The present results did not suggest that BA or CR alone are less effective than their combination, as was observed in some RCTs (McNamara \& Horan, 1986; Taylor \& Marshall, 1977). A previously conducted pairwise meta-analysis (Cuijpers, Cristea et al., 2019) reported a moderate effect size of $g=0.46(0.01$ to 0.91$)$ in favor of CBT in comparison to CR. We also found a moderate effect size between CBT and CR, $d=0.75$ (0.01 to 1.51 ), in a pairwise comparison, although our network results do not provide evidence of such difference. In the network meta-analysis, when also indirect effects were taken into 
account, the effect size decreased to $d=-0.13(-0.62$ to 0.36$)$, meaning no evidence of a difference. Since the two studies providing the direct effects for this comparison had a substantial risk of bias, these results need to be considered with caution. It may be that CBT is indeed superior to CR only as previous research suggests, but the current evidence does not allow for such conclusions and it seems that neither of the conditions is superior to the other. Studies of higher quality are needed to investigate how CR alone really performs.

We found significant inconsistency in the loop among CBT, CR and WL. The inconsistency factor for this loop, i.e., the difference between direct and indirect evidence, was 1.55 (0.29 to 2.80). One reason behind this inconsistency may be the suboptimal quality of the small number of included studies investigating the comparison between $\mathrm{CR}$ and $\mathrm{WL}$, as it was resolved in our first sensitivity analysis (when removing studies with high risk of bias). These studies (i.e., Taylor \& Marshall, 1977; Wilson et al., 1983) were both evaluated to be of high risk of bias, showed huge effects for the given comparison (2.18 [0.82 to 3.54], 1.61 [0.50 to 2.72], respectively) and included small numbers of participants (14 and 17, respectively). In the sensitivity analysis with only low risk of bias studies, also the CR arm was removed due to high risk of bias in all the studies focusing on this condition. Therefore, caution must be taken when making conclusions about the effectiveness of $\mathrm{CR}$ and more research is needed to examine this issue.

Our findings were largely replicated in our sensitivity analyses. When including only the BATD type of BA, the BA intervention was superior to the full CBT. The studies investigating this type of BA (i.e., Gawrysiak et al., 2009; McIndoo et al., 2016; Nasrin et al., 2017) have all considerably small sample sizes (30,30 and 36 participants, respectively), and two out of the three studies (Gawrysiak et al., 2009; McIndoo et al., 2016) were evaluated as having high risk of bias. It is hence possible that the effect of BA was overestimated in this 
analysis. Nevertheless, potentially differential effectiveness of various types of BA warrants further investigation.

The results of the study should be considered in the light of its limitations. First, the evidence from the loop involving CBT, CR and WL was highly inconsistent. In addition, considerably low number of studies examined CR, those which examined it had a high risk of bias and the direct comparison of $\mathrm{CR}$ and CAU was missing completely. Hence, better quality studies, especially high-quality studies that test the components of CBT in head-tohead fashion, are necessary to examine the effectiveness of these conditions. Second, the $95 \%$ CIs of the $I^{2}$ statistic were often very wide and may imply that large heterogeneity among the studies may exist. Yet, the included studies showed only moderate heterogeneity. Third, some studies used additional techniques on top of the ones core to $\mathrm{CR}$ and BA, which may have contributed to the heterogeneity of the psychotherapies examined, and deviations could have occurred from the intended intervention, which was difficult to account for. Notwithstanding, all psychotherapies involved followed an accepted manual for given approach and additional techniques represented only a minority of the time the therapist spent with the participant. Fourth, our analysis did not find any evidence of a difference among the conditions regarding tolerability. The operationalization of tolerability, which was only a proxy, might have contributed to this fact. In addition, our examination of tolerability covered solely the topic of dropout from study, but not from the intervention. Thus, further investigation needs to be conducted to address this question. Fifth, our database was updated in January 2020, we could have therefore missed relevant studies published during the year 2020. Sixth, our inclusion was limited to studies with individual face-to-face format; thus, differential effects of the active conditions in other formats still have to be examined. Finally, a large number of studies was deemed to be at a high risk of bias, and there was evidence of PB-SSE in the comparison of CBT and WL. Nevertheless, our sensitivity and meta-regression analyses 
provided similar results in studies with low risk of bias and there was no evidence of PB-SSE in the comparison of active conditions.

Future research should focus on investigating the effectiveness of the CR condition in comparison to $\mathrm{CBT}$ and BA. Moreover, the results of our sensitivity analyses call for future work towards identifying the most effective approach to delivering BA. Furthermore, the concept of tolerability should be approached as a study, but also treatment dropout, to allow us to identify treatments where a higher number of participants receives an adequate dose of the intervention. Finally, although our approach enabled the optimal use of all available data, our findings still refer to the average effectiveness and tolerability of the treatments at hand. Important predictors of effectiveness and tolerability for individual patients should thus be identified by means of an individual patient data network meta-analysis (IPD-NMA; Debray et al., 2015; Tomlinson et al., 2020).

The current study suggests that CR or BA alone, and their combination (CBT) can be effective interventions in comparison to WL and CAU in the treatment of adult depression. Moreover, in line with the common-factor model, there was no evidence of a difference in effectiveness among these three active interventions. However, the quality of evidence for performance of $\mathrm{CR}$ only was low and our results do not allow us to draw any firm conclusions about the effectiveness of CR alone.

Our findings suggest that administering CBT and BA only may lead to comparable clinical outcomes. This may be true also about CR alone, although more research is needed on this topic. Hence, attention should be also paid to patient's and therapist's preferences and to predictors of differential effectiveness yet to be identified in future research. On top of enhanced personalization of treatment, our results could also contribute to its easier implementation and dissemination. Accessibility of psychotherapy may profit from our findings in settings with no availability of a psychotherapist or other CBT-trained 
professional. For example, it has been suggested that BA can be effectively delivered by lay counsellors (Chowdhary et al., 2016; Patel et al., 2017) or via internet-based interventions (Arjadi et al., 2018); based on that, we might also expect that it can be successfully administered by trained non-specialized helpers, such as public health nurses. Moreover, training in BA or its involvement into self-help interventions may require less time and resources than full CBT. In conclusion, given the suggested comparable effectiveness of the included treatments, preference, predictors of effectiveness or accessibility can inform collaborative decision-making when choosing the treatment for an individual patient. 


\section{References}

(* - included in the meta-analysis)

Arjadi, R., Nauta, M. H., Scholte, W. F., Hollon, S. D., Chowdhary, N., Suryani, A. O., ... \& Bockting, C. L. (2018). Internet-based behavioural activation with lay counsellor support versus online minimal psychoeducation without support for treatment of depression: a randomised controlled trial in Indonesia. The Lancet Psychiatry, 5(9), 707-716.

Azam, K., Serfaty, M., King, M., Martin, S., Strydom, A., Parkes, C., \& Hassiotis, A. (2012). The development of manualised cognitive behaviour treatment for adults with mild intellectual disability and common mental disorders. Psychiatrike= Psychiatriki, 23(2), 109-116.

*Beach, S. R., \& O'Leary, K. D. (1992). Treating depression in the context of marital discord: Outcome and predictors of response of marital therapy versus cognitive therapy. Behavior Therapy, 23(4), 507-528. https://doi.org/10.1016/S0005-7894(05)80219-9

Beck, A. T., Rush, A. J., Shaw, B. F., \& Emery, G. (1979). Cognitive therapy of depression. Guilford Press.

Beck, A. T., Steer, R. A., \& Brown, G. (1996). Beck depression inventory-II. Psychological Assessment. https://doi.org/10.1037/t00742-000

Beck, A. T., Ward, C. H., Mendelson, M., Mock, J., \& Erbaugh, J. (1961). An inventory for measuring depression. Archives of general psychiatry, 4(6), 561-571. https://doi.org/10.1001/archpsyc.1961.01710120031004

Burns, D. D., \& Auerbach, A. (1996). Therapeutic empathy in cognitive-behavioral therapy: Does it really make a difference? In P. M. Salkovskis (Ed.), Frontiers of cognitive therapy (pp. 135-164). Guilford Press.

Burns, D. D. (1999). The feeling good handbook. Plume/Penguin Books. 
*Castonguay, L. G., Schut, A. J., Aikens, D. E., Constantino, M. J., Laurenceau, J. P., Bologh, L., \& Burns, D. D. (2004). Integrative Cognitive Therapy for Depression: A Preliminary Investigation. Journal of Psychotherapy Integration, 14(1), 4-20. https://doi.org/10.1037/1053-0479.14.1.4

Chaimani, A., Higgins, J. P., Mavridis, D., Spyridonos, P., \& Salanti, G. (2013). Graphical tools for network meta-analysis in STATA. PloS one, 8(10), e76654. https://doi.org/10.1371/journal.pone.0076654

Chaimani, A., Salanti, G., Leucht, S., Geddes, J. R., \& Cipriani, A. (2017). Common pitfalls and mistakes in the set-up, analysis and interpretation of results in network meta analysis: what clinicians should look for in a published article. Evidence-based mental health, 20(3), 88-94. https://dx.doi.org/10.1136/eb-2017-102753

*Chowdhary, N., Anand, A., Dimidjian, S., Shinde, S., Weobong, B., Balaji, M., . . Patel, V. (2016). The healthy activity program lay counsellor delivered treatment for severe depression in India: Systematic development and randomised evaluation. British Journal of Psychiatry, 208(4), 381-388. https://doi.org/10.1192/bjp.bp.114.161075

Cipriani, A., Higgins, J. P., Geddes, J. R., \& Salanti, G. (2013). Conceptual and technical challenges in network meta-analysis. Annals of internal medicine, 159(2), 130-137. https://doi.org/10.7326/0003-4819-159-2-201307160-00008

Cohen, J. (1988). Statistical power analysis for the behavioral sciences (2nd ed.). Erlbaum.

Cuijpers, P. (2017). Four decades of outcome research on psychotherapies for adult depression: An overview of a series of meta-analyses. Canadian Psychology/Psychologie Canadienne, 58(1), 7-19. https://doi.org/10.1037/cap0000096

Cuijpers, P., Berking, M., Andersson, G., Quigley, L., Kleiboer, A., \& Dobson, K. S. (2013). A meta-analysis of cognitive-behavioural therapy for adult depression, alone and in 
comparison with other treatments. The Canadian Journal of Psychiatry, 58(7), 376385. https://doi.org/10.1177/070674371305800702

Cuijpers, P., Clignet, F., van Meijel, B., van Straten, A., Li, J., \& Andersson, G. (2011). Psychological treatment of depression in inpatients: a systematic review and metaanalysis. Clinical psychology review, 31(3), 353-360. https://doi.org/10.1016/j.cpr.2011.01.002

Cuijpers, P., Cristea, I. A., Karyotaki, E., Reijnders, M., \& Hollon, S. D. (2019). Component studies of psychological treatments of adult depression: a systematic review and metaanalysis. Psychotherapy Research, 29(1), $15-29$. https://doi.org/10.1080/10503307.2017.1395922

Cuijpers, P., Karyotaki, E., Ciharova, M., Quero, S., Pineda, B., Munoz, R., Struijs, S. Y., Llamas, J., \& Figueroa, C. (2020, January 27). A meta-analytic database of randomised trials on psychotherapies for depression. Open Science Framework. https://osf.io/825c6/

Cuijpers, P., Karyotaki, E., Harrer, M. \& Ebert, D. (2019). Meta-psy. Retrieved from https://evidencebasedpsychotherapies.shinyapps.io/metapsy/

Cuijpers, P., Karyotaki, E., Reijnders, M., Purgato, M., \& Barbui, C. (2018). Psychotherapies for depression in low-and middle-income countries: a meta-analysis. World Psychiatry, 17(1), 90-101. https://doi.org/10.1002/wps.20493

Cuijpers, P., Noma, H., Karyotaki, E., Cipriani, A., \& Furukawa, T. A. (2019). Effectiveness and acceptability of cognitive behavior therapy delivery formats in adults with depression: a network meta-analysis. JAMA psychiatry, 76(7), 700-707. https://doi.org/10.1001/jamapsychiatry.2019.0268

Cuijpers, P., Noma, H., Karyotaki, E., Vinkers, C. H., Cipriani, A. \& Furukawa, T. A. (2020). A network meta-analysis of the effects of psychotherapies, pharmacotherapies and 
their combination in the treatment of adult depression. World Psychiatry, 19(1), 92-107. https://doi.org/10.1002/wps.20701

Cuijpers, P., Quero, S., Noma, H., Ciharova, M., Miguel, C., Karyotaki, E., Cipriani, A., Cristea, I. \& Furukawa, T. A. (in press). Psychotherapies for depression: a network meta-analysis covering efficacy, acceptability and long-term outcomes of all main treatment types. World Psychiatry.

Cuijpers, P., van Straten, A., Warmerdam, L., \& Andersson, G. (2008). Psychological treatment of depression: a meta-analytic database of randomized studies. $B M C$ psychiatry, 8(1), 1-6. https://doi.org/10.1186/1471-244X-8-36

*de Groot, M., Shubrook, J. H., Hornsby, W. G., Pillay, Y., Mather, K. J., Fitzpatrick, K., ... \& Saha, C. (2019). Program ACTIVE II: outcomes from a randomized, multistate community-based depression treatment for rural and urban adults with type 2 diabetes. Diabetes care, 42(7), 1185-1193. https://doi.org/10.2337/dc18-2400

Debray, T. P., Moons, K. G., van Valkenhoef, G., Efthimiou, O., Hummel, N., Groenwold, R. H., ... \& GetReal Methods Review Group. (2015). Get real in individual participant data (IPD) meta-analysis: a review of the methodology. Research synthesis methods, 6(4), 293-309. https://doi.org/10.1002/jrsm.1160

*Desautels, C., Savard, J., Ivers, H., Savard, M. H., \& Caplette-Gingras, A. (2017). Treatment of depressive symptoms in patients with breast cancer: A randomized controlled trialcomparing cognitive therapy and bright light therapy. Health Psychology, 37(1), 1-13. https://doi.org/10.1037/hea0000539

*Dimidjian, S., Hollon, S. D., Dobson, K. S., Schmaling, K. B., Kohlenberg, R. J., Addis, M. E., . . Jacobson, N. S. (2006). Randomized trial of behavioral activation, cognitive therapy, and antidepressant medication in the acute treatment of adults with major 
depression. Journal of Consulting and Clinical Psychology, 74(4), 658-670. https://doi.org/10.1037/0022-006x.74.4.658

Doering, L. V., Chen, B., Cross, R., Magsarili, M. C., Nyamathi, A., \& Irwin, M. R. (2013). Early cognitive behavioral therapy for depression after cardiac surgery. The Journal

of cardiovascular nursing, 28(4), $370-379$. https://doi.org/10.1097/JCN.0b013e31824d967d

Efthimiou, O., Debray, T. P., van Valkenhoef, G., Trelle, S., Panayidou, K., Moons, K. G., ... \& GetReal Methods Review Group. (2016). GetReal in network meta-analysis: a review of the methodology. Research Synthesis Methods, 7(3), 236-263. https://doi.org/10.1002/jrsm.1195

Efthimiou, O., \& White, I. R. (2020). The dark side of the force: Multiplicity issues in network meta-analysis and how to address them. Research Synthesis Methods, 11(1), 105-122. https://doi.org/10.1002/jrsm.1377

Egger, M., Smith, G. D., Schneider, M., \& Minder, C. (1997). Bias in meta-analysis detected by a simple, graphical test. $B m j, 315(7109), \quad 629-634$. https://doi.org/10.1136/bmj.315.7109.629

*Ekers, D., Richards, D., McMillan, D., Bland, J. M., \& Gilbody, S. (2011) Behavioural activation delivered by the nonspecialist: Phase II randomised controlled trial. British Journal of Psychiatry, 198(1), 66-72. https://doi.org/10.1192/bjp.bp.110.079111

Ekers, D., Webster, L., Van Straten, A., Cuijpers, P., Richards, D., \& Gilbody, S. (2014). Behavioural activation for depression; an update of meta-analysis of effectiveness and $\begin{array}{lll}\text { sub-group } \quad \text { analysis. PloS } 9(6), & \text { ond00100. }\end{array}$ https://doi.org/10.1371/journal.pone.0100100 
England, M. J., Butler, A. S., \& Gonzalez, M. L. (2015). Psychosocial interventions for mental and substance use disorders: a framework for establishing evidence-based standards. National Academy Press.

*Euteneuer, F., Dannehl, K., Del Rey, A., Engler, H., Schedlowski, M., \& Rief, W. (2017). Immunological effects of behavioral activation with exercise in major depression: an exploratory randomized controlled trial. Translational psychiatry, 7(5), e1132-e1132. https://doi.org/10.1038/tp.2017.76

*Floyd, M., Scogin, F., McKendree-Smith, N. L., Floyd, D. L., \& Rokke, P. D. (2004). Cognitive therapy for depression: A comparison of individual psychotherapy and bibliotherapy for depressed older adults. Behavior Modification, 28(2), 297-318. https://doi.org/10.1177/0145445503259284

*Freedland, K. E., Carney, R. M., Rich, M. W., Steinmeyer, B. C., \& Rubin, E. H. (2015). Cognitive behavior therapy for depression and self-care in heart failure patients a randomized clinical trial. JAMA Internal Medicine, 175(11), 1773-1782. https://doi.org/10.1001/jamainternmed.2015.5220

*Freedland, K. E., Skala, J. A., Carney, R. M., Rubin, E. H., Lustman, P. J., Dávila-Román, V. G., ... \& Hogue, C. W. (2009). Treatment of depression after coronary artery bypass surgery: a randomized controlled trial. Archives of general psychiatry, 66(4), 387-396. https://doi.org/10.1001/archgenpsychiatry.2009.7

*Fuhr, D. C., Weobong, B., Lazarus, A., Vanobberghen, F., Weiss, H. A., Singla, D. R., ... \& Joshi, A. (2019). Delivering the Thinking Healthy Programme for perinatal depression through peers: an individually randomised controlled trial in India. The Lancet Psychiatry, 6(2), 115-127. https://doi.org/10.1016/S2215-0366(18)30466-8

Furukawa, T. A., Suganuma, A., Ostinelli, E. G., \& Cipriani, A. (in press). Dismantling, optimising and personalising internet cognitive-behavioural therapy for depression: A 
systematic review and individual participant data component network metaanalysis. Lancet Psychiatry.

Furukawa, T. A., Reijnders, M., Kishimoto, S., Sakata, M., DeRubeis, R. J., Dimidjian, S., ... \& Lespérance, F. (2020). Translating the BDI and BDI-II into the HAMD and vice versa with equipercentile linking. Epidemiology and psychiatric sciences, 29(24), 1-

13. https://doi.org/10.1017/S2045796019000088

*Gawrysiak, M., Nicholas, C., \& Hopko, D. R. (2009). Behavioral activation for moderately depressed university students: Randomized controlled trial. Journal of counselling psychology, 56(3), 468-475. https://doi.org/10.1037/a0016383

Hamilton, M. (1986). The Hamilton rating scale for depression. In Assessment of depression (pp. 143-152). Springer.

Harrer, M., Cuijpers, P., Furukawa, T.A, \& Ebert, D. D. (2019). Doing Meta-Analysis in $\mathrm{R}: \quad \mathrm{A}$

Hands-on

Guide.

https://bookdown.org/MathiasHarrer/Doing_Meta_Analysis_in_R/

*Hassiotis, A., Serfaty, M., Azam, K., Strydom, A., Blizard, R., Romeo, R., ... \& King, M. (2013). Manualised individual cognitive behavioural therapy for mood disorders in people with mild to moderate intellectual disability: a feasibility randomised controlled trial. Journal of affective disorders, 151(1), 186-195. https://doi.org/10.1016/j.jad.2013.05.076

Hautzinger, M. (2000). Depression im Alter. Ein kognitiv-verhaltenstherapeutisches Gruppenprogramm. Beltz.

Hautzinger, M. (2013). Cognitive behavior therapy for depression: With online materials. Beltz.

Higgins, J. P., \& Green, S. (2011). Cochrane handbook for systematic reviews of interventions. John Wiley \& Sons. 
Higgins, J. P. T., Jackson, D., Barrett, J. K., Lu, G., Ades, A. E., \& White, I. R. (2012). Consistency and inconsistency in network meta-analysis: concepts and models for multi-arm studies. Research Synthesis Methods, 3(2), 98-110. https://doi.org/10.1002/jrsm.1044

Higgins, J. P., Thompson, S. G., Deeks, J. J., \& Altman, D. G. (2003). Measuring inconsistency in meta-analyses. $B M J, 327(7414)$, $557-560$. https://doi.org/10.1136/bmj.327.7414.557

Hutton, B., Salanti, G., Caldwell, D. M., Chaimani, A., Schmid, C. H., Cameron, C., ... \& Mulrow, C. (2015). The PRISMA extension statement for reporting of systematic reviews incorporating network meta-analyses of health care interventions: checklist and explanations. Annals of internal medicine, 162(11), 777-784. https://doi.org/10.7326/M14-2385

Ioannidis, J. P., Patsopoulos, N. A., \& Evangelou, E. (2007). Uncertainty in heterogeneity estimates in meta-analyses. $B M J, 335(7626), \quad 914-916$. https://doi.org/10.1136/bmj.39343.408449.80

*Jacobson, N. S., Dobson, K. S., Truax, P. A., Addis, M. E., Koerner, K., Gollan, J. K., ... \& Prince, S. E. (1996). A component analysis of cognitive-behavioral treatment for depression. Journal of Consulting and Clinical Psychology, 85(1), 26-36. https://doi.org/10.1037/ccp0000151

Jacobson, N. S., Martell, C. R., \& Dimidjian, S. (2001). Behavioral activation treatment for depression: Returning to contextual roots. Clinical Psychology: science and practice, 8(3), 255-270. https://doi.org/10.1093/clipsy.8.3.255

*Jordans, M. J., Luitel, N. P., Garman, E., Kohrt, B. A., Rathod, S. D., Shrestha, P., ... \& Patel, V. (2019). Effectiveness of psychological treatments for depression and alcohol use disorder delivered by community-based counsellors: two pragmatic randomised 
controlled trials within primary healthcare in Nepal. The British Journal of Psychiatry, 215(2), 485-493. https://doi.org/10.1192/bjp.2018.300

*Kanter, J. W., Santiago-Rivera, A. L., Santos, M. M., Nagy, G., López, M., Hurtado, G. D., \& West, P. (2015). A randomized hybrid efficacy and effectiveness trial of behavioral activation for Latinos with depression. Behavior Therapy, 46(2), 177-192. https://doi.org/10.1016/j.beth.2014.09.011

Kroenke, K., Spitzer, R. L., \& Williams, J. B. (2001). The PHQ-9: validity of a brief depression severity measure. Journal of general internal medicine, 16(9), 606-613. https://doi.org/10.1046/j.1525-1497.2001.016009606.x

*Laidlaw, K., Davidson, K., Toner, H., Jackson, G., Clark, S., Law, J., . . . Cross, S. (2008).

A randomised controlled trial of cognitive behaviour therapy vs treatment as usual in the treatment of mild to moderate late life depression. International Journal of Geriatric Psychiatry, 23(8), 843-850. https://doi.org/10.1002/gps.1993

Lejuez, C. W., Hopko, D. R., \& Hopko, S. D. (2001). A brief behavioral activation treatment for depression: Treatment manual. Behavior Modification, 25(2), 255-286. https://doi.org/10.1177/0145445501252005

Lejuez, C. W., Hopko, D. R., LePage, J. P., Hopko, S. D., \& McNeil, D. W. (2001). A brief behavioral activation treatment for depression. Cognitive and Behavioral Practice, 8(2), 164-175. https://doi.org/10.1016/S1077-7229(01)80022-5

Lewinsohn, P. M. (1974). A behavioral approach to depression. Essential papers on depression, 150-172.

*Maldonado, A. L. (1982). Terapia de conducta y depresión: Un análisis experimental de los modelos conductual y cognitivo. Revista de Psicologìa General y Aplicada, 37(1), 3156. 
Martell, C. R., Addis, M. E., \& Jacobson, N. S. (2001). Depression in context: Strategies for guided action. WW Norton \& Co.

Mavridis D. (2019). Network meta-analysis in a nutshell. Evidence-Based Mental Health, 22(3), 100-101. http://dx.doi.org/10.1136/ebmental-2019-300104

Mazzucchelli, T., Kane, R., \& Rees, C. (2009). Behavioral activation treatments for depression in adults: a meta-analysis and review. Clinical Psychology: Science and Practice, 16(4), 383-411. https://doi.org/10.1111/j.1468-2850.2009.01178.x

*McIndoo, C. C., File, A. A., Preddy, T., Clark, C. G., \& Hopko, D. R. (2016). Mindfulnessbased therapy and behavioral activation: A randomized controlled trial with depressed college students. Behaviour Research and Therapy, 77, 118-128. https://doi.org/10.1016/j.brat.2015.12.012

*McNamara, K., \& Horan, J. J. (1986). Experimental construct validity in the evaluation of cognitive and behavioral treatments for depression. Journal of Counseling Psychology, 33(1), 23-30. https://doi.org/10.1037/0022-0167.33.1.23

Moorey, S., \& Greer, S. (2002). Cognitive behaviour therapy for people with cancer. Oxford University Press.

*Nakagawa, A., Mitsuda, D., Sado, M., Abe, T., Fujisawa, D., Kikuchi, T., . . . Ono, Y. (2017).Effectiveness of supplementary cognitive-behavioral therapy for pharmacotherapy resistant depression: A randomized controlled trial. Journal of clinical psychiatry, 78(8), 1126-1135. https://doi.org/10.4088/JCP.15m10511

*Nasrin, F., Rimes, K., Reinecke, A., Rinck, M., \& Barnhofer, T. (2017). Effects of brief behavioural activation on approach and avoidance tendencies in acute depression: Preliminary findings. Behavioural and Cognitive Psychotherapy, 45(1), 58-72. https://doi.org/10.1017/S1352465816000394 
Orsini, N., Bottai, M., Higgins, J., \& Buchan, I. (2006). Heterogi: Stata module to quantify heterogeneity in a meta-analysis. Statistical Software Components. http://econpapers.repec.org/software/bocbocode/s449201.htm

*Pace, T. M., \& Dixon, D. N. (1993). Changes in depressive self-schemata and depressive symptoms following cognitive therapy. Journal of Counseling Psychology, 40(3), 288- 194. https://doi.org/10.1037/0022-0167.40.3.288

*Patel, V., Weobong, B., Weiss, H. A., Anand, A., Bhat, B., Katti, B., ... \& Vijayakumar, L. (2017). The Healthy Activity Program (HAP), a lay counsellor-delivered brief psychological treatment for severe depression, in primary care in India: a randomised controlled trial. The Lancet, 389(10065), 176-185. https://doi.org/10.1016/S0140-6736(16)31589-6

*Pecheur, D. R., \& Edwards, K. J. (1984). A comparison of secular and religious versions of cognitive therapy with depressed Christian college students. Journal of Psychology and Theology, 12(1), 45-54. https://doi.org/10.1177/009164718401200106

*Power M. J., \& Freeman, C. (2012). A randomized controlled trial of IPT versus CBT in primary care: With some cautionary notes about handling missing values in clinical trials. Clinical psychology \& psychotherapy, 19(2), 159-169. https://doi.org/10.1002/cpp.1781

*Propst, L. R., Ostrom, R., Watkins, P., Dean, T., \& Mashburn, D. (1992). Comparative efficacy of religious and nonreligious cognitive-behavioral therapy for the treatment of clinicaldepression in religious individuals. Journal of Consulting and Clinical Psychology, 60(1), 94-103. https://doi.org/10.1007/BF01173648

Radloff, L. S. (1977). The CES-D scale: A self-report depression scale for research in the general population. Applied psychological measurement, 1(3), 385-401. https://doi.org/10.1177/014662167700100306 
*Raue, P. J., Sirey, J. A., Dawson, A., Berman, J., \& Bruce, M. L. (2019). Lay-delivered behavioral activation for depressed senior center clients: Pilot RCT. International journal of geriatric psychiatry, 34(11), 1715-1723. https://doi.org/10.1002/gps.5186

Rehm, L. P. (1984). Self-management therapy for depression. Advances in Behaviour Research and Therapy, 6(2), 83-98. https://doi.org/10.1016/0146-6402(84)90004-3

*Richards, D. A., Ekers, D., McMillan, D., Taylor, R. S., Byford, S., Warren, F. C., ... \& O'Mahen, H. (2016). Cost and Outcome of Behavioural Activation versus Cognitive Behavioural Therapy for Depression (COBRA): a randomised, controlled, noninferiority trial. The Lancet, 388(10047), 871-880. https://doi.org/10.1016/S01406736(16)31140-0

*Rief, W., Bleichhardt, G., Dannehl, K., Euteneuer, F., \& Wambach, K. (2018). Comparing the efficacy of CBASP with two versions of CBT for depression in a routine care center: A randomized clinical trial. Psychotherapy and psychosomatics, 87(3), 164-178. https://doi.org/10.1159/000487893

Rybarczyk, B., Gallagher-Thompson, D., Rodman, J., Zeiss, A., Gantz, F. E., \& Yesavage, J. (1992). Applying cognitive-behavioral psychotherapy to the chronically ill elderly: Treatment issues and case illustration. International Psychogeriatrics, 4(1), 127-140. https://doi.org/10.1017/S1041610292000954

Safran, J. D., \& Segal, Z. V. (1990). Interpersonal Process in Cognitive Therapy. Basic Books.

*Savard, J., Simard, S., Giguere, I., Ivers, H., Morin, C. M., Maunsell, E., . . Marceau, D. (2006). Randomized clinical trial on cognitive therapy for depression in women with metastatic breast cancer: psychological and immunological effects. Palliative $\&$ Supportive Care, 4(3), 219-237. https://doi.org/10.1017/S1478951506060305 
Salanti, G. (2012). Indirect and mixed-treatment comparison, network, or multiple-treatments meta-analysis: many names, many benefits, many concerns for the next generation evidence synthesis tool. Research synthesis methods, 3(2), 80-97. https://doi.org/10.1002/jrsm.1037

Salanti, G., Ades, A. E., \& Ioannidis, J. P. (2011). Graphical methods and numerical summaries for presenting results from multiple-treatment meta-analysis: an overview and tutorial. Journal of clinical epidemiology,64(2), 163-171. https://doi.org/10.1016/j.jclinepi.2010.03.016

Salanti, G., Higgins, J. P., Ades, A. E., \& Ioannidis, J. P. (2008). Evaluation of networks of randomized trials. Statistical methods in medical research,17(3), 279-301. https://doi.org/10.1177/0962280207080643

*Scott, A. I., \& Freeman, C. P. (1992). Edinburgh primary care depression study: treatment outcome, patient satisfaction, and cost after 16 weeks. BMJ, 304(6831), 883-887. https://doi.org/10.1136/bmj.304.6831.883

*Scott, C. S., Scott, J. L., Tacchi, M. J., \& Jones, R. H. (1994). Abbreviated cognitive therapy for depression: a pilot study in primary care. Behavioural and Cognitive Psychotherapy, 22(1), 57-64. https://doi.org/10.1017/S1352465800011802

Scott, C., Tacchi, M. J., Jones, R., \& Scott, J. (1997). Acute and one-year outcome of a randomised controlled trial of brief cognitive therapy for major depressive disorder in primary care. The British Journal of Psychiatry, 171, 131-134.

*Serfaty, M. A., Haworth, D., Blanchard, M., Buszewicz, M., Murad, S., \& King, M. (2009). Clinical effectiveness of individual cognitive behavioral therapy for depressed older people in primary care: a randomized controlled trial. Archives of general psychiatry, 66(12), 1332-1340. https://doi.org/10.1001/archgenpsychiatry.2009.165 
*Serfaty, M. A., King, M., Nazareth, I., Moorey, S., Aspden, T., Mannix, K., ... \& Jones, L. (2019). Effectiveness of cognitive-behavioural therapy for depression in advanced cancer: CanTalk randomised controlled trial. The British Journal of Psychiatry, 216(4), 213-221. https://doi.org/10.1192/bjp.2019.207

Skala, J. A., Carney, R. M., \& Freedland, K. E. (2005). Heart disease. Hogrefe.

StataCorp. (2019). Stata Statistical Software: Release 16. StataCorp LLC.

*Taylor, F. G., \& Marshall, W. L. (1977). Experimental analysis of a cognitive-behavioral therapy for depression. Cognitive Therapy and Research, 1(1), 59-72. https://doi.org/10.1007/BF01173505

*Taylor, C. B., Conrad, A., Wilhelm, F. H., Strachowski, D., Khaylis, A., Neri, E., ... \& Spiegel, D. (2009). Does improving mood in depressed patients alter factors that may affect cardiovascular disease risk?. Journal of psychiatric research, 43(16), 1246-

1252. https://doi.org/10.1016/j.jpsychires.2009.05.006

*Teichman, Y., Bar-el, Z., Shor, H., Sirota, P., \& Elizur, A. (1995). A comparison of two modalities of cognitive therapy (individual and marital) in treating depression. Psychiatry, 58(2), 136-148. https://doi.org/10.1080/00332747.1995.11024721

*Thomas, S. A., Drummond, A. E., Lincoln, N. B., Palmer, R. L., das Nair, R., Latimer, N. R., ... \& Cooper, C. L. (2019). Behavioural activation therapy for post-stroke depression: the BEADS feasibility RCT. Health technology assessment (Winchester, England), 23(47), 1-176. https://doi.org/10.3310/hta23470

Thompson, L. W. (1996). Cognitive-behavioral therapy and treatment for late-life depression. The Journal of clinical psychiatry. 57(Suppl 5), 29-37.

*Thompson, L. W., Gallagher, D., \& Breckenridge, J. S. (1987). Comparative effectiveness of psychotherapies for depressed elders. Journal of Consulting and Clinical Psychology, 55(3), 385-390. https://doi.org/10.1037//0022-006x.55.3.385 
Tomlinson, A., Furukawa, T. A., Efthimiou, O., Salanti, G., De Crescenzo, F., Singh, I., \& Cipriani, A. (2020). Personalise antidepressant treatment for unipolar depression combining individual choices, risks and big data (PETRUSHKA): rationale and protocol. Evidence-Based Mental Health, 23(2), 52-56. http://dx.doi.org/10.1136/ebmental-2019-300118

*Tovote, K. A., Fleer, J., Snippe, E., Peeters, A., Emmelkamp, P. M. G., Sanderman, R., . . . Schroevers, M. J. (2014). Individual mindfulness-based cognitive therapy and cognitive behavior therapy for treating depressive symptoms in patients with diabetes: Results of a randomized controlled trial. Diabetes Care, 37(9), 24272434. https://doi.org/10.2337/dc13-2918

Uphoff, E., Pires, M., Barbui, C., Barua, D., Churchill, R., Cristofalo, D., ... \& Siddiqi, N. (2020). Behavioural activation therapy for depression in adults with noncommunicable diseases. Cochrane Database of Systematic Reviews, (8). https://doi.org/10.1002/14651858.CD013461.pub2

Yost, E. B., Beutler, L. E., Corbishley, M. A., Allender, J. R., \& Dryden, W. (1987). Group Cognitive Therapy: A Treatment Approach for Depressed Older Adults. Journal of Cognitive Psychotherapy, 1(3), 200-200.

Wahl, I., Löwe, B., Bjorner, J. B., Fischer, F., Langs, G., Voderholzer, U., ... \& Rose, M. (2014). Standardization of depression measurement: a common metric was developed for 11 self-report depression measures. Journal of clinical epidemiology, 67(1), 73-86. https://doi.org/10.1016/j.jclinepi.2013.04.019

Wampold, B. E., \& Imel, Z. E. (2015). The great psychotherapy debate: The research evidence for what works in psychotherapy. Routledge.

White, I. R. (2015). Network meta-analysis. The Stata Journal, 15(4), 951-985. https://doi.org/10.1177/1536867X1501500403 
Wilkinson, P. (1997). Cognitive therapy with elderly people. Age and Ageing, 26, 53-58.

*Wilson, P. H., Goldin, J. C., \& Charbonneau-Powis, M. (1983). Comparative efficacy of behavioral and cognitive treatments of depression. Cognitive Therapy and Research, 7(2), 111-124. https://doi.org/10.1007/BF01190064

Whisman, M. A. (1993). Mediators and moderators of change in cognitive therapy of depression. Psychological Bulletin, 114(2), 248-265.

https://doi.org/10.1037/0033-2909.114.2.248

*Wright, J. H., Wright, A. S., Albano, A. M., Basco, M. R., Goldsmith, L. J., Raffield, T., \& Otto, M. W. (2005). Computer-assisted cognitive therapy for depression: maintaining efficacy while reducing therapist time. American Journal of Psychiatry, 162(6), 11581164. https://doi.org/10.1176/appi.ajp.162.6.1158

Zigmond, A. S., \& Snaith, R. P. (1983). The hospital anxiety and depression scale. Acta psychiatrica scandinavica, 67(6), 361-370.

https://doi.org/10.1111/j.1600-0447.1983.tb09716.x

Table 1

Pairwise Meta-analyses of CBT, $C R$ and $B A^{a}$

\begin{tabular}{l|lllll}
\hline Condition comparison & No. & SMD $(95 \% \mathrm{CI})$ & $I^{2}$ Statistic $(95 \% \mathrm{CI})$ & tau $^{2}$ & Egger Test $P$ Value \\
\hline CBT vs CR & 2 & $0.75(-0.01$ to 1.51$)$ & $13(\mathrm{NC})$ & 0.04 & $b$ \\
CBT vs BA & 6 & $-0.03(-0.19$ to 0.13$)$ & $0(0-61)$ & 0.00 & .53 \\
CBT vs WL & 15 & $1.07(0.81$ to 1.33$)$ & $53(0-72)$ & 0.13 & .001 \\
CBT vs CAU & 12 & $0.48(0.28$ to 0.68$)$ & $51(0-73)$ & 0.06 & .70 \\
CR vs BA & 4 & $0.13(-0.41$ to 0.66$)$ & $16(0-73)$ & 0.05 & .30 \\
CR vs WL & 2 & $1.84(0.98$ to 2.70$)$ & $0(\mathrm{NC})$ & 0.00 & $b$ \\
BA vs WL & 5 & $1.52(1.13$ to 1.91$)$ & $0(0-64)$ & 0.00 & .22 \\
BA vs CAU & 8 & $0.49(0.27$ to 0.71$)$ & $52(0-76)$ & 0.04 & .31 \\
\hline
\end{tabular}

Note. Abbreviations (alphabetical): BA: Behavioral activation; CAU: Care as usual; CBT: Cognitive behavior therapy; CR:

Cognitive restructuring; NC: Not calculated; WL: Waitlist condition.

A positive (negative) SMD favors the first (second) treatment in each comparison.

${ }^{a}$ No studies were available for the comparison of CR and CAU, and CAU and WL.

${ }^{b}$ This cannot be calculated when the number of studies is smaller than 3 . 


\section{Figure 1. Network Plot}

Note. Abbreviations (alphabetical): BA: Behavioral activation; CAU: Care as usual; CBT:

Cognitive behavior therapy; CR: Cognitive restructuring; WL: Waitlist.

Table 2

\section{Estimated Relative
Cognitive Behavioral $^{\prime}$}

$-0.13(-0.62$ to 0.36$)[-0$

$\overline{-0.08(-0.26 \text { to } 0.10)[-0}$

$1.07(0.87$ to 1.26$)[0.6($

0.44 (0.28 to 0.60$)[-0.0$

Note. The treatme

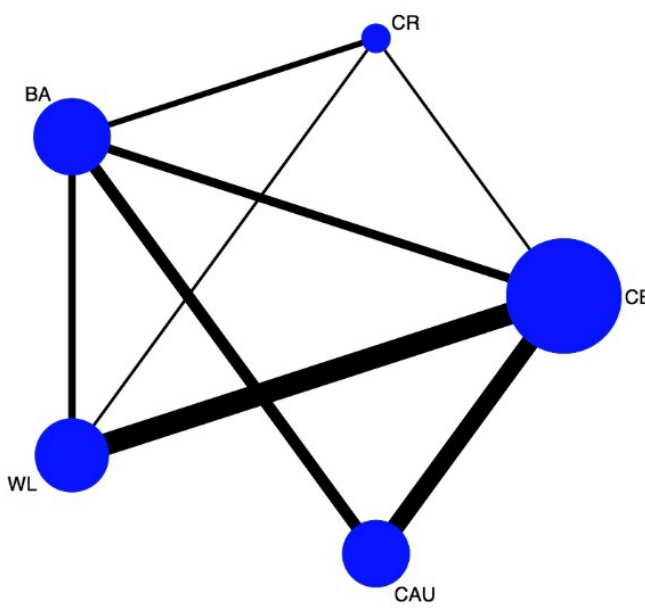

Diagonal) and Tolerability (above Diagonal)

\begin{tabular}{l|l|l}
\hline .05 to 30.80$]$ & $0.80(0.04$ to 18.08$)[0.03$ to 20.52$]$ & $0.96(0.76$ to 1.21$)[0.63$ to 1.46$]$ \\
\hline $\mathbf{n}$ & $0.66(0.42$ to 1.05$)[0.37$ to 1.20$]$ & $0.01(0.04$ to 22.81$)[0.04$ to 25.90$]$ \\
\hline 65 to 1.64$]$ & Waiting List & \\
\hline 06 to 0.99$]$ & $-0.63(-0.87$ to -0.38$)$ to $[-1.12$ to -0.14$]$ & Care as Usual \\
\hline
\end{tabular}

ers underneath the diagonal denote standardized mean differences with 95\%

CIs and 95\% PIs for every specific contrast between the nodes. A number larger than zero favors the column-defining treatment of the cell. Numbers above the diagonal show relative risks for tolerability with $95 \%$ CIs and 95\% PIs. A relative risk larger than 1 favors the row-defining treatment.
(A) Effectiveness
(B) Tolerability

Figure 2. Estimated Effectiveness (in SMD) and Tolerability (in RR) of CBT, CR, BA and WL versus CAU. 
Reference treatment: CAU Note.

Treatment Effect

SMD $(95 \% \mathrm{Cl})$

Abbreviations Reference treatment: CAU

Treatment Effect

RR $(95 \% \mathrm{Cl})$

(alphabetical):

BA: Behavioral activation; CBT: Cognitive behavioral

therapy; CR:

Cognitive

CR

restructuring; SMD: Standardized mean difference;

WL:

$\longrightarrow 0.57(0.08,1.07)$

WL

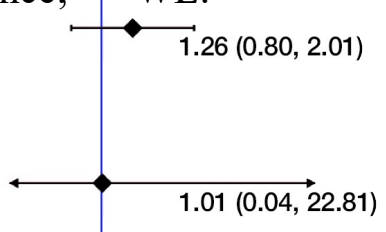

BA

$\mapsto \quad 0.52(0.34,0.71) \quad$ The

$\mathrm{CR}$

reference group

for both the effectiveness (A) and tolerability (B) plots

is CAU.

CBT

$\multimap \quad 0.44(0.28,0.60)$

CBT

Table 3

WL

$-0.63(-0.87,-0.38)$

$\mathrm{BA}$

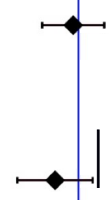

$0.96(0.76,1.21)$

Ranking of All Treatments after Network Meta-analysis, according to the Surface under the Cumulative

\begin{tabular}{|c|c|c|}
\hline vours Care as Usual 1 & Favours Intervention & Favours Care as Usual \\
\hline Condition & \begin{tabular}{l|l} 
& Effectiveness, \% \\
\end{tabular} & Tolerability, $\%$ \\
\hline Cognitive Behavioral Therapy & 62.4 & 45.0 \\
\hline Cognitive Restructuring & 81.5 & 49.8 \\
\hline Behavioral Activation & 80.8 & 19.3 \\
\hline Waiting List & 0.0 & 81.6 \\
\hline Care as Usual & 25.3 & 54.3 \\
\hline
\end{tabular}

Note. Abbreviations (alphabetical): BA: Behavioral activation; CBT: Cognitive behavioral therapy; CR:

Cognitive restructuring.

Table 4

Long-term Effects of CBT, CR, BA and Control Conditions

\begin{tabular}{|c|c|c|c|c|c|c|c|c|c|c|c|c|}
\hline & \multicolumn{5}{|c|}{ Network meta-analyses } & \multicolumn{7}{|c|}{ Pairwise meta-analyses } \\
\hline Contrast $^{a}$ & SMD & $95 \% \mathrm{CI}$ & & $95 \% \mathrm{PI}$ & No. & SMD & $95 \% \mathrm{C}$ & & $I^{2}$ & $95 \% \mathrm{CI}$ & $\operatorname{tau}^{2}$ & $\overline{\text { Egger }(p)}$ \\
\hline CBT vs CR & -0.31 & -0.91 & to & $-0.98 \sim 0.36$ & 1 & -0.02 & -1.17 & to & $a$ & $a$ & $a$ & $\bar{a}$ \\
\hline CBT & -0.03 & $\begin{array}{l}0.29 \\
-0.17\end{array}$ & to & $-0.31 \sim 0.26$ & 3 & 0.02 & $\begin{array}{l}1.13 \\
-0.16\end{array}$ & to & 0 & $0-73$ & 0.00 & .21 \\
\hline BA & & 0.12 & & & & & 0.20 & & & & & \\
\hline CBT & 1.13 & 0.37 & to & $0.30 \sim 1.95$ & 0 & & & & & & & \\
\hline $\begin{array}{l}\text { WL } \\
\text { CBT vs }\end{array}$ & 0.37 & $\begin{array}{l}1.88 \\
0.26\end{array}$ & to & $0.10 \sim 0.63$ & 21 & 0.36 & 0.24 & to & 33 & $0-60$ & 0.02 & .59 \\
\hline CAU & & 0.47 & & & & & 0.48 & & & & & \\
\hline CR vs BA & 0.29 & -0.30 & to & $-0.37 \sim 0.94$ & 3 & 0.41 & -0.29 & to & 25 & $0-79$ & 0.10 & .53 \\
\hline CR vs WL & 1.44 & $\begin{array}{l}0.87 \\
0.49\end{array}$ & to & $0.43 \sim 2.45$ & 0 & & 1.10 & & & & & \\
\hline $\mathrm{CR}$ & 0.68 & $\begin{array}{l}2.38 \\
0.08\end{array}$ & to & $0.01 \sim 1.35$ & 0 & & & & & & & \\
\hline CAU & & 1.28 & & & & & & & & & & \\
\hline BA vs WL & 1.15 & 0.41 & to & $0.34 \sim 1.96$ & 1 & 1.18 & 0.48 & to & $a$ & $a$ & $a$ & $a$ \\
\hline BA vs & 0.39 & $\begin{array}{l}1.89 \\
0.26\end{array}$ & to & $0.11 \sim 0.67$ & 7 & 0.41 & $\begin{array}{l}1.88 \\
0.28\end{array}$ & to & 31 & $0-70$ & 0.01 & .27 \\
\hline
\end{tabular}




\begin{tabular}{l|lll|l|}
$\mathrm{CAU}$ \\
$\mathrm{WL}$ & & 0.52 & & \\
$\mathrm{CAU}$ & -0.76 & -1.51 & to & $-1.58 \sim 0.06$ \\
& & -0.01 & & \\
\hline
\end{tabular}

Note. Abbreviations (alphabetical): BA: Behavioral activation; CAU: Care as usual; CBT: Cognitive behavior therapy; CR: Cognitive restructuring; NC: Not calculated; WL: Waitlist condition.

${ }^{a}$ This cannot be calculated when the number of studies is smaller than 3 . 